\title{
DeskJockey: Exploiting Passive Surfaces to Display Peripheral Information
}

\author{
Ryder Ziola, Melanie Kellar, and Kori Inkpen \\ Dalhousie University, Faculty of Computer Science \\ Halifax, NS, Canada \\ \{ziola, melanie, inkpen\}@cs.dal.ca
}

\begin{abstract}
This paper describes DeskJockey, a system to provide users with additional display space by projecting information on passive physical surfaces in the environment. The current DeskJockey prototype utilizes a projected desk and allows information to be moved easily between active and passive displays using a world-in-miniature interaction metaphor. A four-week, in-situ field study was conducted to compare usage of DeskJockey with typical multiple monitor use. The results revealed potential for utilizing passive physical surfaces in this manner and demonstrated that this type of display space has distinctive affordances and benefits which enhance traditional display space.
\end{abstract}

Keywords: Augmented desk, multiple monitors, peripheral information, display space management, user interaction.

\section{Introduction}

Traditional single-user, single-monitor workstations are often enhanced by adding more displays, increasing the available screen real estate. Studies of the use of multiple monitors have revealed that additional displays are often treated as a discrete place to view output, rather than being used to enlarge the user's active working area $[6,7]$. Additionally, secondary monitors are often used to monitor information in the periphery, providing "instant access to a resource in a known location in peripheral vision" [6]. Given that continuity of screen space is not essential when monitoring peripheral information, the opportunity to make these dedicated locations resident on a different medium is appealing.

Currently, the division between the physical desk and the computer monitor is defined by digital or physical content; a physical clock sits on our physical desk, while virtual clocks are often placed on our computer monitor. In this paper, we examine augmented surfaces for individual users to extend their workspace onto passive physical surfaces in the environment. Our work blurs the boundary between physical and virtual, allowing active and passive media to coexist. The aim is not to turn the desk into an active display; a myriad of properties (resolution, orientation, interaction strategies) currently make it a poor substitute for a monitor for many tasks. Instead, we can capitalize on the passive nature of traditional desks to display passive digital information - information primarily used for reference, to provide context, or 
to monitor something peripheral or unconnected to the central task The challenge therefore is to design a desk which can house real paper next to digital calendar information, digital sticky notes next to the (physical) telephone, while keeping users subtly informed about their world. While explicitly not attempting to augment reality, this system would at least allow the virtual to bump up against the physical more casually.

The overall goal of this research is to increase users' available display space by utilizing physical surfaces in the environment to casually display peripheral information (See Figure 1). This offloads the need to display peripheral information on the primary monitor(s). A secondary goal of our research was to determine whether a straightforward implementation of an augmented surface, where most of the interaction occurred on the desktop monitor, would provide benefits for individual users. While Rekimoto \& Saitoh's Augmented Surfaces [11], provided a spatially continuous workspace to move digital information among various devices in a collaborative environment (including tables and walls), their architecture was inherently complex. Instead we are examining a more straightforward implementation based on off-the-shelf components, where most of the interaction occurs on the desktop monitor. Finally, we were interested in observing how people make use of the augmented surface, including what types of objects they put on the surface, how persistent these objects were, and how often people interacted with the objects.

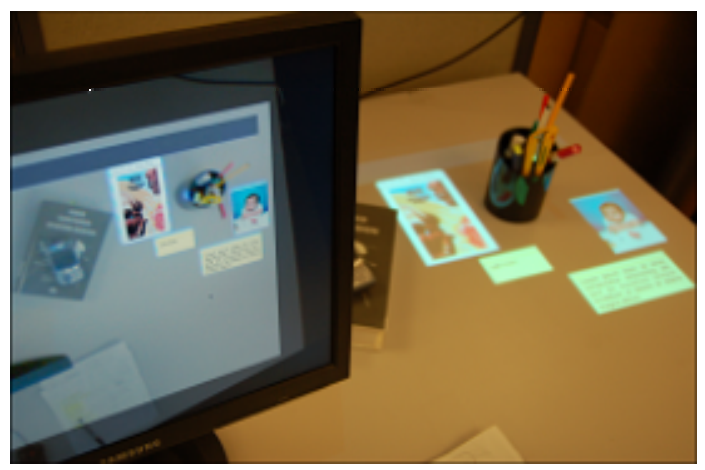

Fig. 1. The DeskJockey System where digital information can be placed on a physical desk, amongst other physical artifacts that reside in this space

Paper Overview. This paper explores the design of augmented surfaces for individual users to extend their workspace onto passive physical surfaces in the environment. We describe the development of DeskJockey, a system which utilizes a projected display to place peripheral information on a physical desk (Figure 1). In this system, digital information can easily be "dropped" onto the desk for peripheral monitoring, or can be brought back into focus on the users' primary display when additional interaction or information is required. We report on a 4-week field study that observed usage of DeskJockey, compared to a multi-monitor setup, to identify how people would utilize such a system during real day-to-day activities. 


\section{Related Work}

\subsection{Augmented Desks}

The idea of displaying digital information on a desk is not new. Much work has been done on enhanced desk systems, including the DigitalDesk [14], InteractiveDesk [1] and metaDESK [12]. These approaches were primarily aimed at either augmenting the physical desk with digital information [14], or interacting with virtual objects using physical artifacts $[1,12]$ Our approach is different in that we are not attempting to merge virtual and physical representations, instead we are taking advantage of the available space to place various types of digital information into the physical environment and enrich our periphery.

Rekimoto and Saitoh [11] introduced the notion of augmented surfaces in which tables and walls could be used to provide an extended desktop for portable computers. The motivation for this work was to support co-located collaboration and enable information to move freely between surfaces in the environment (hyperdragging). However, users are required to interact using the cursor on a tabletop display. This can be awkward, particularly if the table is cluttered. While similar to the ideas presented in this paper, Rekimoto and Saitoh's augmented surfaces are intended to be manifestations of an extended desktop which supports active interaction. Rather than creating a new workspace to replace or compete with the main monitor as the primary locus of interaction, we intend to supplement it with a physically large area used strictly for secondary output where users interact with the peripheral information from the main monitor.

\subsection{Peripheral and Ambient Information}

Information can be considered peripheral if it is not necessary for the current task, but may be of interest to the user nonetheless. Information monitoring tasks form a large subset of peripheral activities and centre on the problem of notification: how to minimise distraction while informing the user of potentially important or interesting changes in the system's state. This category includes tasks such as being notified of incoming e-mails, being aware of an upcoming meeting, or seeing which friends are logged into their instant messaging application. As we are interested in providing support for peripheral information monitoring, the mechanisms necessary for performing it should be examined.

Investigations into the requirements for a successful peripheral interface focus on the goal of notifying the user of a change in the information being monitored without allowing it to hinder the current task. Maglio \& Campbell [9] performed three studies to compare various methods of peripherally presenting text, including a number of variations on a scrolling ticker, fading text, and visual and accompanying auditory feedback. It was found that continuous motion, in the form of a scrolling ticker, was both detrimental to performance of the primary task and unreliable at notifying users of the peripheral information. Discrete motion, active only when new information is presented, was found to be most effective. Exploring this idea further, Bartram et al. [2] studied the ability of Moticons - icons with simple motion - to notify users of 
changes. They reported that motion is detected better than changes in colour or shape, especially in the periphery of the user's vision, and is well suited to notification.

A subset of peripheral information, ambient information, can be identified by its intended subtlety. It is meant to implicitly communicate information not necessarily relevant to the task at hand through environmental cues and is not intended as a means of explicit notification. The ambientROOM [8] aims to create an environment that interfaces with digital information, attempting to engage the human ability to process background information. For example, the current activity-level of a loved-one is displayed as ripples projected on the ceiling of the room and the volume of a background soundtrack of nature sounds can roughly signal quantities (such as incoming e-mail). These fully-fledged ambient environments require dedicated physical objects or displays and naturally come with high infrastructure requirements.

As we are interested in supporting ambient uses, the design requirements for ambient information prove useful. Mankoff et al. [10] developed heuristics for the evaluation of ambient displays that help to inform us of the affordances a system must offer to be compatible with ambient use. In particular, two of their heuristics stand out as being specifically relevant to the design of a system capable of supporting ambient uses, rather than the design of the ambient application itself:

- perpipherality of display: it can be easily monitored while being unobtrusive.

- easy transition to more in-depth information: it is necessary to be able to easily engage the system, converting the interaction from passive to active.

A system intended for use in part as an ambient display should accommodate these properties. Unlike previous work that used dedicated displays or specifically designed embodied devices [16], DeskJockey can place ambient information on available physical surfaces. This provides unobtrusive monitoring and facilitates interactions as users shift from passive to active.

\section{Augmented Surfaces for Individual Workspaces}

We have designed a system that allows peripheral and ambient information to be dispersed throughout the environment by projecting virtual objects onto physical surfaces. This idea can be utilized on any workspace surface, making the tabletop or a nearby wall a potential repository for digital information (see Figure 2). Additionally, a completely clear space is not necessary (and is not practical), as the system can project arbitrary shapes and sizes on whatever space is available on the physical surface. Additionally, if a top-projected system is used, the system can display objects on top of physical objects in the environment. For example, if a user's desk was cluttered with piles of paper, they could turn the top paper over or place a blank sheet on the top. The system could then project onto the stack, thereby utilizing that space. When projecting on a wall, this problem is less likely to be encountered.

By default, a projected display surface is passive. While vision tracking can be used to provide various augmented reality features such as object tracking, occlusion detection, and active linking, previous research has shown that manipulating objects 


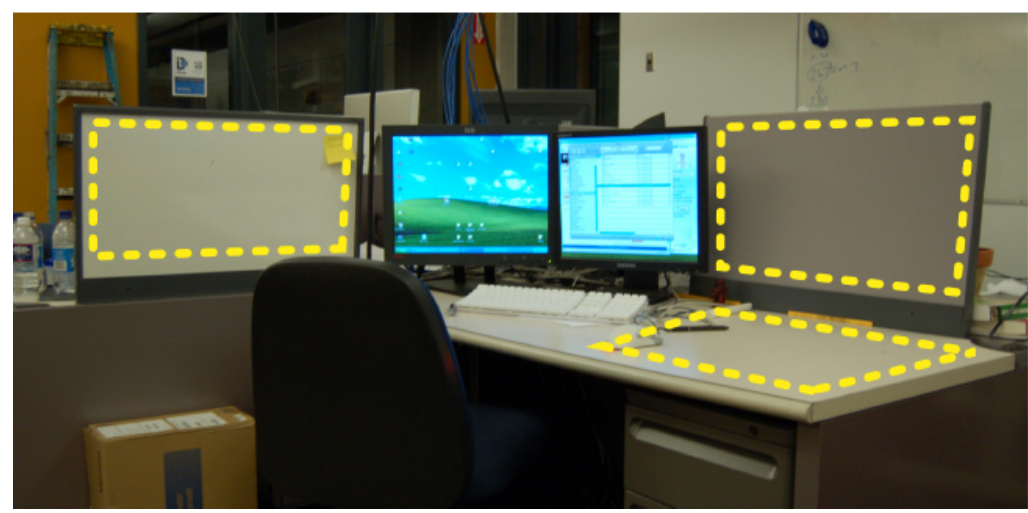

Fig. 2. Any physical surface in the environment can be utilized to display peripheral information

on extended surfaces is challenging [13]. In particular, it is difficult to detect discrete events with small-scale hand movements, particularly because users frequently make movements like this in the course of their work [13]. Wilson [15] noted that computer vision techniques are often "unnecessarily complex and inferior" when emulating a single Windows cursor on a table.

Our aim was to provide lightweight access to digital objects on peripheral display spaces and to enable users to easily place, retrieve, and interact with objects. Additionally, many monitoring tasks serve as a gateway into an active task; as a result it was necessary to accommodate a passive surface as the starting point for an active task. We chose to initially investigate a system whereby most interactions with virtual objects on the passive surface would take place using the desktop computer, using standard GUI interactions.

Two types of interaction are required to support passive projected displays. First, a navigation mechanism is needed to traverse, move, and place objects on the display. Second, techniques are needed to perform detailed interaction with the objects themselves (i.e., editing a post-it note).

We identified several methods of navigating virtual objects on the projected display, employing varying degrees of abstraction with respect to interaction with the projected display. Depending on the interaction model used, detailed interaction with objects on the projected display can either occur in-place (i.e., on the projected surface itself), or on the primary monitor. Interaction in-place is intuitive; however, traditional desktop environments are better suited for standard types of interaction.

The first technique identified, direct traversal, simply treats the projected surface as an extension of the workstation's primary display (See Figure 3a). With this technique, the mouse travels between the primary display and the projected display, similar to a traditional multi-monitor configuration as well as Rekimoto and Saitoh's hyperdragging technique [11]. The second technique, cursor jump, allows the cursor to jump between displays by pressing a mouse button or key-combination (see Figure 3b), similar to Benko and Feiner's multi-monitor mouse [3]. The third technique, bring here, involves having all of the objects on the projected display zoom to the primary display for interaction (see Figure 3c), similar to the Mac OS X's Exposé 


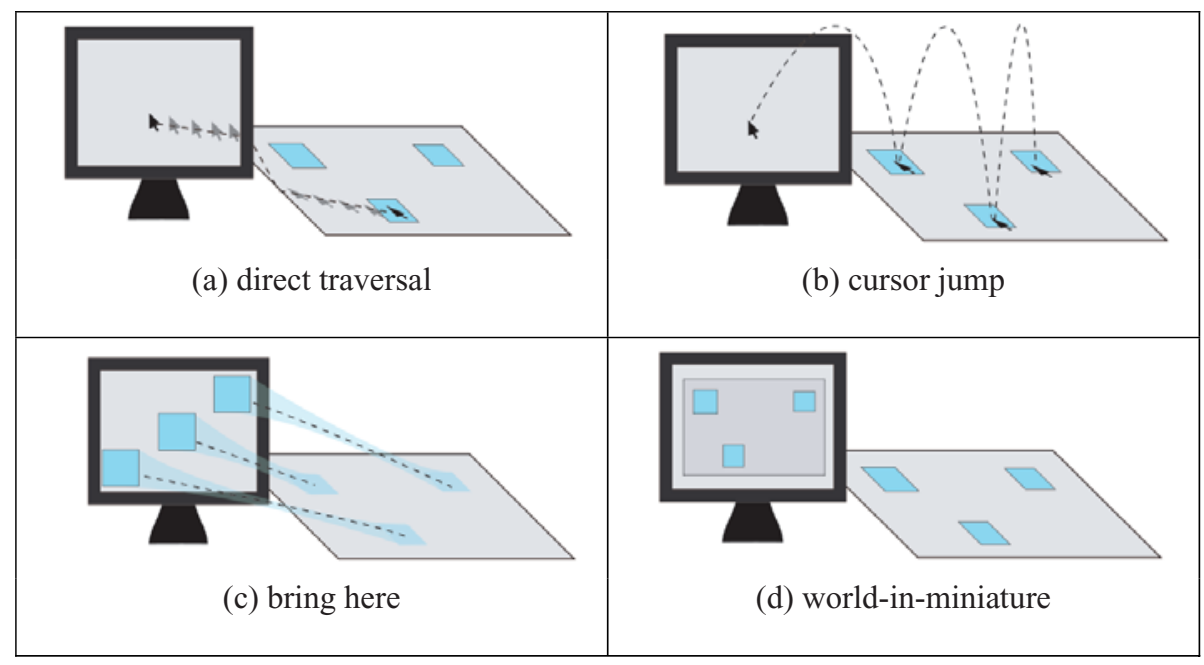

Fig. 3. Proposed interaction models to navigate and interact with virtual objects on the physical surface

feature [5]. The fourth technique, world-in-miniature, involves interacting with a representation of the projected display on the primary monitor (see Figure 3d).

\section{DeskJockey}

We designed and implemented DeskJockey, a prototype augmented surface system to further explore system design issues, and to investigate how people would utilize augmented surfaces as part of their regular, day-to-day work environment.

\subsection{Physical Setup}

The standard physical set-up of our DeskJockey prototype includes a workstation with a single 19" monitor (1280 x 960 resolution), a $100 \mathrm{~cm} \times 150 \mathrm{~cm}$ desk serving as the extended workspace, and a projector, projecting an image to the right of the keyboard. The projected display is given a black background so that virtual objects on the desk appeared cropped, with nothing being projected on the unused parts of the desk. In order to facilitate the placement of virtual objects with respect to real objects, a simple webcam ( $640 \times 480$ resolution) is mounted overhead to provide a background to the system, comprised of real-time video of the desk. This setup could also incorporate a multi-monitor setup if desired.

\subsection{WiM Interaction Model}

We chose to utilize the World-in-Miniature (WiM) interaction model for DeskJockey because it provides quick access to the table and its contents, it takes advantage of the rich interaction capabilities of the desktop, and it does not interfere with interactions on the primary display. Real-time video of the desk (obtained from the overhead 
camera) was used as the background for the WiM. The low resolution image obtained from the camera was sufficient for distinguishing physical artifacts on the table; however, details on the virtual objects were hard to discern. We therefore chose to draw the icons representing the virtual images over top of the video of the table. This required that the icons be aligned perfectly with the video beneath them. There are too many factors - camera, projector, and table orientation - to achieve the precision necessary purely through mechanical alignment. To compensate for these effects, a perspective transform was applied to the incoming video (See Figure 4).

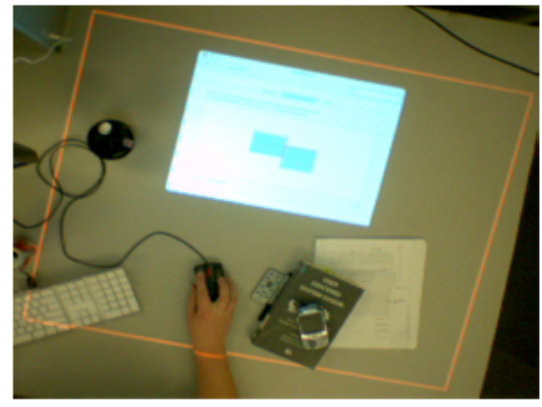

(a) Video Before Correction

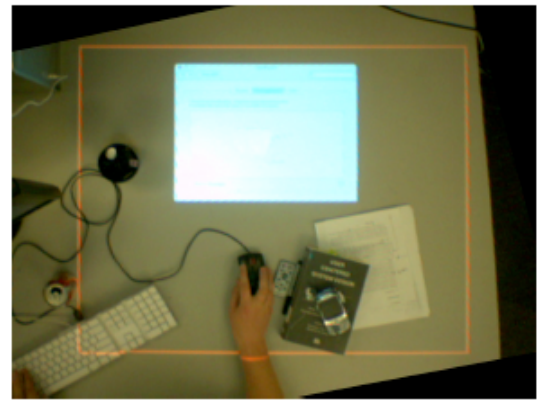

(b) Video After Correction

Fig. 4. A perspective transformation is applied to the incoming video from the web cam to help align overlaid images

DeskJockey's WiM is activated by moving the cursor to a hotspot that extends across the bottom of the primary display (Figure 5). The bottom of the display provides a natural mapping in that moving down off the monitor moves the cursor down onto the desk. When the cursor is moved off the bottom of the screen, a transition to view the WiM is triggered. To minimize accidental triggering, the transition does not occur if the cursor is moving slower than a specified threshold.

Animated feedback is provided to signify that the user is transitioning to the WiM. The WiM view is mapped to a surface that lies perpendicular to the bottom of the primary monitor's current contents. The screen simulates a $90^{\circ}$ rotation effect, moving the primary monitor's current contents up and off the screen (i.e., imagine a rotating cube), replacing it with a miniature view of the objects on the desk. Conversely, once viewing the WiM, moving up off the top of the screen triggers the reverse transition and the primary monitor returns to its normal view.
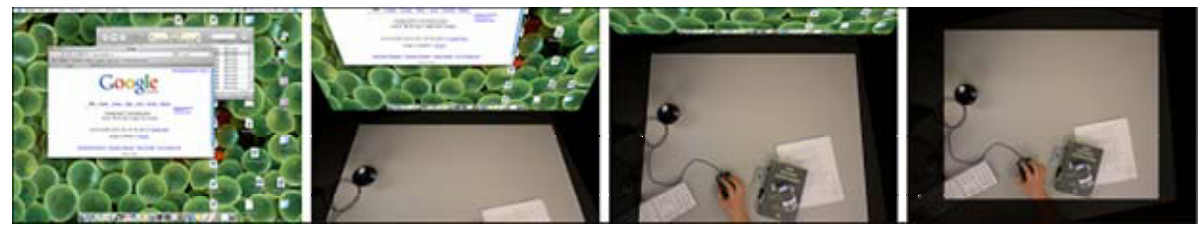

Fig. 5. The visual transition to the world-in-miniature 


\subsection{Navigating the WiM}

Once the WiM has been activated, the user is presented with a representational view of the desk as shown in Figure 6. The view is centered on the projected area of the desk, in the same orientation as the projector. One consequence of working with a WiM rather than the objects themselves is the loss of context provided by the real desk. Without a mechanism for directly seeing how the extended workspace corresponds to the real desk, orientation is difficult to understand and avoiding collisions with physical artifacts on the desk becomes difficult. Real-time video of the desk provides the background for the WiM which enables the user to easily place virtual objects with respect to real objects on the desk.

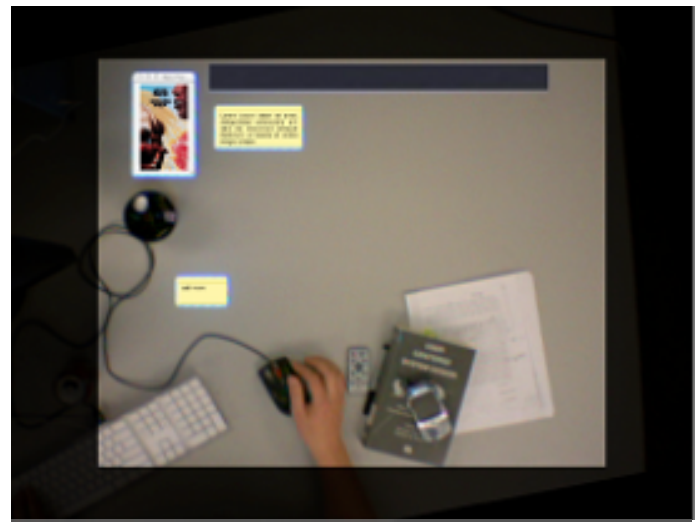

Fig. 6. The WiM offers a real-time view of the desk to facilitate placement and interaction with virtual objects

Any window in the workspace can be added to the desk by dragging it to the WiM. This is achieved by dragging the window down to the hotspot on the bottom of the screen. Similarly, any virtual object can be removed from the desk and returned to the primary display by dragging it up off of the WiM. Each virtual object on the desk is represented as an icon in the WiM. In an attempt to make the metaphor as robust as possible, icons are shown as a screenshot of the object they represent, scaled to correct proportions. The icons can be dragged around the world-in-miniature to move the virtual object on the desk. When the cursor moves over an icon, it glows yellow, along with the object on the desk, to help reinforce the correspondence between the icons and the objects they represent.

\subsection{Interacting with Virtual Objects on the Desk}

Although the main purpose of the system is for passive information monitoring, interaction with items on the desk is nonetheless a requirement. Interaction with virtual objects in DeskJockey takes place on the primary monitor and is activated by clicking on the object in the WiM. This moves the object from the desk to the foreground of the primary monitor for interaction (see Figure 7). To return to the $\mathrm{WiM}$, the user clicks anywhere off of the object or presses escape. 


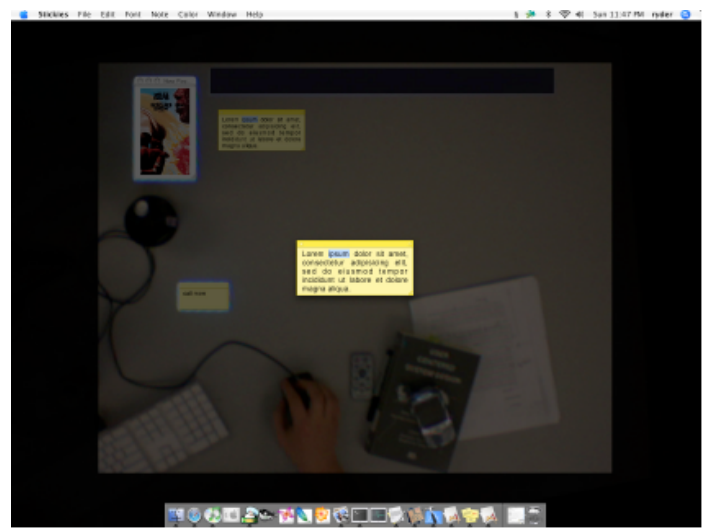

Fig. 7. Objects on the table are brought to the primary display for manipulation

The state transition diagram in Figure 8 illustrates the actions to transition to/from normal desktop interaction, to the WiM, to object manipulation.

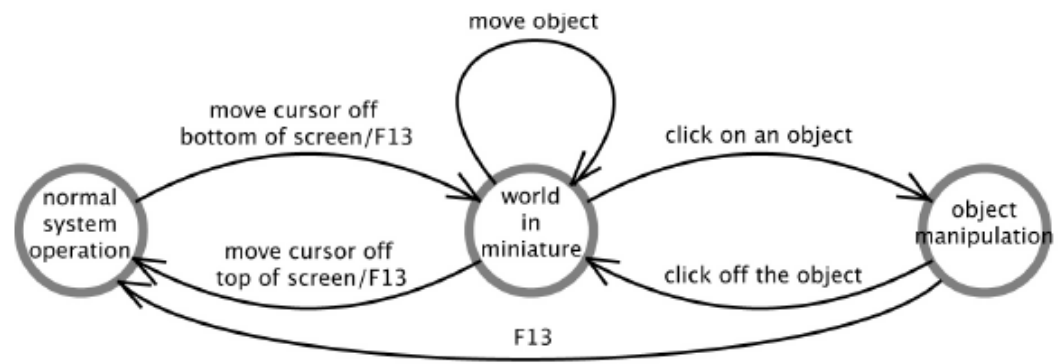

Fig. 8. State transition diagram illustrating progression from normal operation, to the WiM, to interaction

\subsection{Sample Applications}

Although the DeskJockey prototype can display any window on the projected surface, some applications are better suited to being placed on a passive display than others. We have selected, modified, or developed several applications to illustrate specific uses (Figure 9 provides a screen shot of some of these applications).

- Stickies. A program for placing Post-It style notes on your desktop.

- Pics. A program to display an image or PDF without a border or titlebar, at an arbitrary size

- Clutter. An open-source program for displaying cover art for the .mp3 currently playing and making casual piles of covers that can be selected to play the album [4]. This allows cover art for the current song to sit on the desk and digital albums to be scattered on the desk much like their physical counterparts. 
- Widget Viewer. A generic wrapper to display a Mac OS X Dashboard widget as a window that can be placed on the desk such as a calendar widget, a weather widget, and a widget that represents new emails as flowers in a vase. Many of these small programs are designed to monitor information or status.

- $S k y$. A daylight monitor was created as an example of the ambient information potential of DeskJockey. Sky loads an image from a local outdoor webcam, calculates the average colour of the sky and displays a large block of that colour with the texture of the sky from the camera. During the day, it roughly communicates if the sky is blue or grey and becomes dark as the sun sets.

\section{DeskJockey Field Study}

Although many additional features could be added to DeskJockey to increase the novelty of the system, we first wanted to examine the potential of augmented surfaces for everyday use. A field study was undertaken to explore how DeskJockey would be used in a natural environment and how DeskJockey differed from a more traditional dual monitor environment. We were also interested in gathering feedback on the WiM interface. Although augmented surfaces have been proposed by others in past work, to our knowledge no one has investigated sustained, real-use of such a system.

Five computer science students ( 1 female) were recruited to take part in a 4-week study. During the first two weeks, participants were assigned to one of two conditions: DeskJockey or dual monitors. At the end of the second week, participants switched conditions (condition order was counterbalanced). Four out of five of the participants had previous experience using a multi-monitor system. Both conditions were run using an Apple PowerBook or a PowerMac G5. The dual monitor condition utilized two 19" monitors. In both conditions, logging software was installed on the participant's computer. This software recorded screen captures of both displays every 30 minutes for the duration of the study (see Figure 10 for an example screen shot).

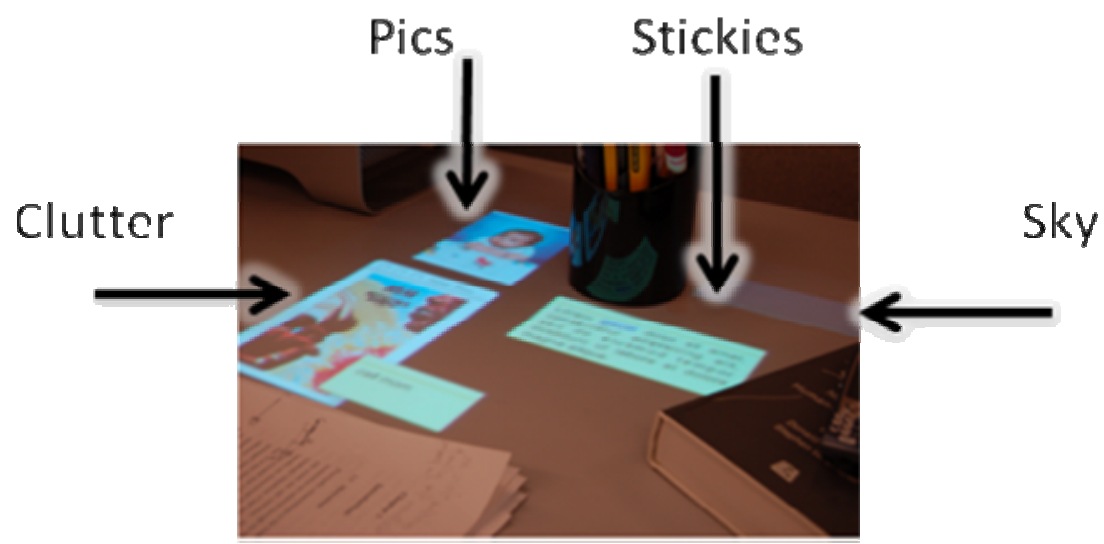

Fig. 9. Some of the supplemental applications developed for DeskJockey 
Upon completion of the study, participants took part in a 30 minute interview where they were asked to describe their experiences with both display conditions. A researcher selected a small number of screen shots to be used during the interview. Using the screen shots as a guide, participants were asked to describe the tasks being performed and the applications being used in both displays (primary and projected).

\subsection{Results}

DeskJockey Observations. Throughout the course of the study, we observed a number of applications being placed within the projected display space (Figure 10 shows an example screen shot from the study). Weather widgets were the most commonly reported applications (by all 5 participants), followed by clocks (4/5), a digital light-fixture application that displayed coloured spotlights $(4 / 5)$, pictures $(3 / 5)$, and calendars (3/5). Two participants indicated that they displayed their instant messenger list while others displayed their iTunes player or a transit information widget. One interesting usage was the placement of a participant's del.icio.us cloud on the projected display. This participant remarked that due to the size of the tag cloud it was not feasible to place it on a toolbar or desktop but that DeskJockey provided a "handy off to the side" reference. Although this list is not comprehensive, it clearly indicates that all participants used DeskJockey as part of their daily activities, and provides an indication of the types of applications that participants felt were appropriate for the passive display surface.

All participants expressed overall enthusiasm for DeskJockey, and 4 of the 5 participants reported that it was useful for their daily activities. Participants cited the primary strength of DeskJockey as the ability to use peripheral display space that could not be used otherwise. While many of the applications placed in DeskJockey were also viewed within the dual monitor condition, the DeskJockey environment

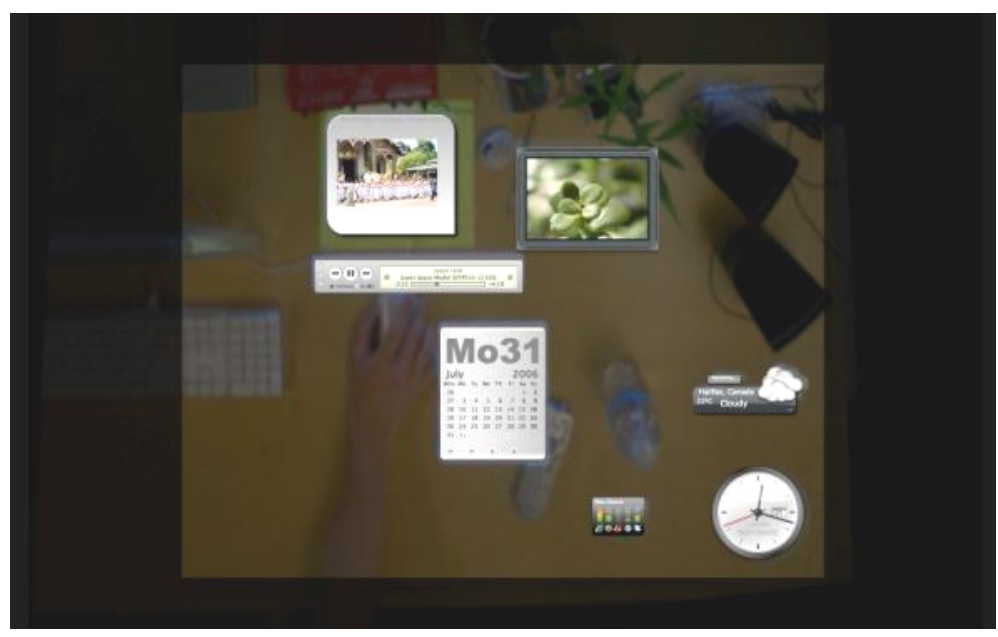

Fig. 10. Example screen shot from the DeskJockey user study showing various applications that were placed on the projected display 
allowed participants to keep these applications open continually throughout the day, without having to overlap windows. One participant reported that he enjoyed the ability to project onto multiple surfaces. His monitor resided on a raised surface onto which he projected his clock and calendar application while the remaining applications were projected on the desk itself. Another participant reported that he liked using DeskJockey for information that he did not want to be a distraction.

The main weakness reported for DeskJockey was the current implementation of the WiM. While the WiM was found to be useful, participants reported they often inadvertently activated the WiM when overshooting the Dock (which was located on the bottom of their screen). Three participants stated they would rather drag applications onto the desk through the right side of the monitor while one reported that he would have preferred a hotkey to toggle to the WiM. Other frustrations related to the prototype nature of DeskJockey including not being able to easily rotate applications (a limitation of the current prototype), problems manipulating objects in the WiM, and web browser windows occasionally disappearing within the DeskJockey environment.

Dual-Monitor Observations. Consistent with previous research, we observed that all participants partitioned their work across the two screens. The primary monitor typically contained an active application and the secondary monitor displayed information that was being monitored (e.g., email and instant messenger) and reference information (e.g., documents, web sites). The biggest strength of the dual monitor setup (in comparison to a single monitor setup) reported by participants was the added screen real estate.

DeskJockey versus Dual Monitor. Participants were asked to describe how they used DeskJockey in comparison with the dual monitor environment. All participants stated they placed peripheral applications that required little interaction in the DeskJockey environment. Many of these applications, such as weather, calendars, and webcam views, were not continually displayed during the dual monitor condition but were instead launched as needed. The secondary display was viewed as an extension of the active workspace where screen real estate is too valuable to "waste" on such applications. One participant stated that that "the second monitor is a place to extend my workspace, while DeskJockey is a place to put peripheral information, [such as] pictures".

\section{Conclusion and Future Work}

In this paper we presented a prototype system called DeskJockey, which capitalizes upon the large size and relatively static layout of a desk to provide additional peripheral display space. DeskJockey uses readily available equipment and requires little infrastructure. DeskJockey's world-in-miniature technique addresses unique interface problems that arise from secondary displays that show content but do not support interaction directly. Our prototype implementation demonstrated that it is feasible to enhance an environment without adding to the active workspace area.

The main contribution of this work is an investigation of augmented surfaces to extend individuals' virtual workspace onto passive surfaces in the environment. 
Virtual objects - such as calendars, to-do lists, reminder notes, or incoming emails can be projected alongside physical objects on the desk or walls. This approach represents a novel way of seeing a projected table as an extension to a traditional workstation, rather than a primary locus of interaction.

Another key contribution is the results from the 4-week in-situ, field study which demonstrate benefits of displaying peripheral information on physical surfaces in the environment. Our participants appreciated the ability to exploit available surfaces that would otherwise be wasted. DeskJockey was found to be fundamentally different than a second monitor; participants displayed different types of information, for longer periods of time, without sacrificing valuable screen real estate. The results also indicated that DeskJockey has strong potential for supporting information monitoring activities, which are becoming an important web and desktop activity.

While the generalizability of our results are limited given the small sample size, we felt that it was important to: (1) evaluate whether or not this type of system would be used for real-world activities, and (2) better understand the types of tasks it would be used to support. Now that we have a better understanding of how the system will likely be used, we can further refine the prototype so that it can be deployed more widely in the future. Additionally, further in-situ evaluations are necessary to determine how usage of the DeskJockey space would evolve over time.

Our current implementation chose a WiM approach, forcing all interaction to take place on the primary monitor. While this approach had merit, participants had difficulty with the interaction used to activate/deactivate the WiM. As such, they expressed frustration and would have preferred more flexibility in the types of interactions (e.g., direct interaction or the ability to use hotkeys). Further examination is needed, particularly when compared to the other interaction models proposed. As we better understand usage of this type of environment, we will be able to explore new interaction techniques for DeskJockey.

\section{Acknowledgements}

We would like to thanks the Canadian Natural Sciences and Engineering Research Council and NECTAR for funding this work. Additional thanks go to members of the EDGE Lab for their assistance and to the participants in our field study.

\section{References}

1. Arai, T., Machii, K., Kuzunuki, S., Shojima, H.: Interactivedesk: a computer-augmented desk which responds to operations on real objects. In: Proceedings of CHI '95, pp. 141142 (1995)

2. Bartram, L., Ware, C., Calvert, T.: Moticons: detection, distraction and task. Int. J. Hum.Comput. Stud. 58(5), 515-545 (2003)

3. Benko, H., Feiner, S.: Multi-monitor mouse. In: Proceedings of CHI '05, pp. 1208-1211 (2005)

4. Clutter, http://http://www.sprote.com/clutter/

5. Exposé, http://www.apple.com/macosx/features/expose/ 
6. Grudin, J.: Partitioning digital worlds: focal and peripheral awareness in multiple monitor use. In: Proceedings of CHI '01, pp. 458-465 (2001)

7. Hutchings, D.R., Smith, G., Meyers, B., Czerwinski, M., Robertson, G.: Display space usage and window management operation comparisons between single monitor and multiple monitor users. In: Proceedings of AVI '04, pp. 32-29 (2004)

8. Ishii, H., et al.: ambientROOM: Integrating ambient media with architectural space. In: Proceedings of CHI '98, pp. 173-174 (1998)

9. Maglio, P., Campbell, C.S.: Tradeoffs in displaying peripheral information. In: Proceedings of CHI '00, pp. 241-248 (2000)

10. Mankoff, J., et al.: Heuristic evaluation of ambient displays. In: Proceedings of CHI '03, pp. 169-76 (2003)

11. Rekimoto, J., Saitoh, M.: Augmented surfaces: A spatially continuous workspace for hybrid computing environments. In: Proceedings of CHI'99, pp. 378-385 (1999)

12. Ullmer, B., Ishii, H.: The metadesk: Models and prototypes for tangible user interfaces. In: Proceedings of UIST '97, pp. 223-232 (1997)

13. Voida, S., Podlasec, M., Kjeldsen, R., Pinhanez, C.: A study on the manipulation of 2D objects in a projector/camera-based augmented reality environment. In: Proceedings of CHI 2005, pp. 610-611 (2005)

14. Wellner, P.: Interacting with paper on the DigitalDesk. Communications of the ACM 36(7), 87-96 (1993)

15. Wilson, A.: PlayAnwhere: A compact interactive tabletop projection-vision system. In: Proceedings of UIST 2005, pp. 83-92 (2005)

16. Wisneski, C., et al.: Ambient Displays: Turning Architectural Spaces into an Interface between People and Digital Information. In: Streitz, N.A., Konomi, S., Burkhardt, H.-J. (eds.) CoBuild 1998. LNCS, vol. 1370, pp. 22-32. Springer, Heidelberg (1998) 